\title{
Avaliação da Prevalência de Nefropatia pelo Uso de Contraste Iodado em Pacientes Diabéticos no Serviço de Hemodinâmica do Hospital Universitário Sul Fluminense (HUSF), de Vassouras-RJ
}

\author{
Mariane de Moura Oliveira \\ Discente do Curso de Medicina da Universidade Severino Sombra, \\ USS, Vassouras/RJ \\ Fábio Cavalheira Pássaro \\ Discente Curso de Medicina da Universidade Severino Sombra, \\ USS, Vassouras/RJ \\ Marcelo Augusto Paiva Rio de Oliveira \\ Médico Nefrologista do Hospital Universitário sul Fluminense, \\ HUSF, Vassouras/RJ \\ Marcio José Montenegro da Costa \\ Médico Hemodinamicista do Hospital Universitário Sul \\ Fluminense, HUSF, Vassouras/RJ \\ Wiliam Faviere \\ Médico Coordenador de Pesquisa do Hospital Universitário Sul \\ Fluminense, HUSF, Vassouras/RJ \\ wfav@uol.com.br
}

Resumo: A nefropatia por contraste iodado (NCI) consiste numa complicação importante e freqüente nos centros de hemodinâmica. Objetivo: Definir a prevalência de NCI nos pacientes diabéticos que passaram por procedimentos que utilizaram contraste iodado no laboratório de Hemodinâmica do HUSF, no periodo de Junho a Agosto, de 2009. Metodologia: Preenchimento de ficha de identificação dos pacientes admitidos no setor, coleta de amostra sanguínea para detecção de glicemia e creatinina sérica, e aferição da pressão arterial. Resultados: Durante o período pesquisado, foram estudados 245 pacientes com idade entre 33 a 90 anos. Dos 150 individuos que foram acompanhados, sendo que 35 pacientes desenvolveram NCI (27\%). Evidenciou-se 38 eram portadores de DM (29,23\%), e destes, 8 individuos (21,05\%) desenvolveram NCI. Conclusão: Como visto nos resultados expostos, $21,05 \%$ dos pacientes diabéticos desenvolveram NCI, configurando incidência média 11 vezes maior quando comparado à população geral.

Palavras-chave: Nefropatia. Contraste iodado. Diabetes Mellitus. 


\title{
Evaluation of the Incidence of Contrast-induced Nephropathy in Diabetic Patients at the Hemodynamic Laboratory from Hospital Universitário Sul Fluminense, Vassouras-RJ
}

\begin{abstract}
Contrast induced nephropathy consists (CIN) is a frequent and important complication at centers of hemodynamic. Objective: To define the incidence of CIN in diabetic patients who underwent procedures using iodinate contrast at the hemodynamic lab from HUSF, between June and August, 2009. Methods: An identification form was filled with information of all patients that were admitted; a blood sample was collected to detect both serum creatinine and glucose, and blood pressure was measured. Results: During the survey, we studied 245 patients aged 33-90 years. At ambulatory, 150 patients were followed, where 35 patients developed CIN (27\%). This study demonstrate that 38 patients were diabetic (29.23\%), and 8 of them (21.05\%) developed CIN. Conclusion: As exposed above, 21,05\% of the diabetic patients developed CIN, which is 11 times higher when compared do general population.
\end{abstract}

Keywords: Nephropathy. Iodinate contrast. Diabetes Mellitus.

\section{Introdução}

A nefropatia por contraste iodado (NCI) consiste numa complicação importante e bastante freqüente nos centros de hemodinâmica. Resulta muitas vezes em insuficiência renal aguda (IRA) iatrogênica, sendo a terceira causa de IRA em pacientes hospitalizados, acarretando em maior tempo de internação hospitalar.

Define-se NCI, na maioria das vezes, pelo aumento da creatinina sérica em mais de $25 \%$ do valor de base, ou aumento absoluto de $0,5 \mathrm{mg} / \mathrm{dL}$, após 48 a $72 \mathrm{~h}$ da administração do contraste iodado endovenoso, na ausência de outras causas. O pico de creatinina sérica é atingido em 4 a 5 dias, geralmente retornando ao nível basal após 1 semana.

O uso do contraste radiológico tem sido empregado desde 1923 como método diagnóstico e terapêutico. Os primeiros relatos de seus efeitos adversos surgiram em 1941, e aumentaram a partir de 1954. Atualmente, a NCI constitui um tema de grande interesse em pesquisas médicas, tanto para elucidar precisamente sua patogênese, quanto para estabelecer medidas terapêuticas e profiláticas eficazes.

Dados epidemiológicos demonstram maior incidência em indivíduos que apresentam comorbidades. Ganha destaque dentre os diversos fatores de risco, o diabetes mellitus (DM), visto que consiste em uma patologia crônica, que provoca lesões renais de forma lenta e insidiosa. Indivíduos portadores de DM e insuficiência renal leve apresentam fluxo sanguíneo renal e resposta hormonal reduzida aos agentes vasoativos, que associado ao uso do contraste contribui para o aumento da prevalência da NCI nesse grupo. 
A profilaxia dessa entidade ainda permanece controversa. Diversos estudos têm sido realizados com o intuito de reduzir a incidência da NIC, e evitar perda da função renal.

\section{Objetivo}

Definir a incidência da NCI nos pacientes diabéticos que passaram por procedimentos que utilizaram contraste iodado no laboratório de Hemodinâmica no Hospital Universitário Sul Fluminense (HUSF), no período de Junho a Agosto, de 2009.

\section{Metodologia}

Elaboramos uma ficha a ser preenchida no laboratório de Hemodinâmica previamente ao procedimento, contendo dados gerais do paciente. Além dessa ficha, constava um pedido de exame de sangue para detecção da glicemia de jejum e creatinina sérica; e uma solicitação de retorno para acompanhamento ambulatorial do estado geral e função renal dentro de 3 a 7 dias após o procedimento.

Foram considerados hipertensos todos aqueles que relataram conhecimento de sua condição, bem como aqueles que a desconheciam, porém apresentaram níveis tensionais superiores a $140 \mathrm{mmHg}$ para pressão arterial sistólica, E/OU superior a $90 \mathrm{mmHg}$ para pressão arterial diastólica. Para tanto, recomendou-se coleta da amostra sanguínea após a aferição da PA, evitando-se, assim, falso-positivos.

Em relação ao DM, consideramos indivíduos diabéticos aqueles que relataram ser portadores da doença, ou que tiveram a glicemia de jejum dosada antes do início do procedimento, e esta evidenciou níveis superiores a $126 \mathrm{mg} / \mathrm{dL}$ - o tempo estipulado para jejum foi de $12 \mathrm{~h}$.

O paciente deveria retornar ao ambulatório de Nefrologia HUSF dentro de 3 a 7 dias para acompanhamento ambulatorial, onde foi colhida uma nova amostra sanguínea para dosagem da creatinina sérica após o procedimento.

Os valores de creatinina sérica pré e pós-procedimento foram comparados: consideramos que houve desenvolvimento de NCI em todos aqueles que apresentaram elevação da creatinina sérica maior que $25 \%$ ou aumento de $0,5 \mathrm{mg} / \mathrm{dL}$ do valor dosado inicialmente.

A pesquisa teve duração de 92 dias, sendo realizada de $1^{\circ}$ de Junho a 31 de Agosto de 2009.

\section{Resultados}

Durante o período pesquisado, foram estudados 245 pacientes com idade entre 33 a 90 anos. Não retornaram para avaliação pós-procedimento 115 pacientes (47\%).

Dos 150 indivíduos que foram acompanhados em consulta ambulatorial, 35 pacientes desenvolveram NCI (27\%). 
Evidenciou-se 38 eram portadores de DM (29,23\%), e destes, 8 indivíduos $(21,05 \%)$ desenvolveram NCI. Ressaltamos que todos os diabéticos analisados eram também hipertensos.

\section{Discussão}

Segundo o KDOQI, a NCI é identificada pela alteração da função renal (taxa de filtração glomerular ou nível de creatinina sérica) e pelo período de tempo em que essas alterações se instalam. Portanto, inexiste uma definição padrão para essa condição. De modo geral, para efeitos de estudos e pesquisas, considera-se o aumento da creatinina sérica em $0,5 \mathrm{mg}$ / dL (44mmol/L) ou o aumento de 25 a $50 \%$ do valor basal, ou ainda a redução de $25 \%$ da taxa de filtração glomerular (TFG), lembrando que faz-se necessário a exclusão de outras causas que podem levar à IRA. A maioria dos estudos demonstram que essas alterações são evidentes 48 a $72 \mathrm{~h}$ após a administração do contraste, com um pico na creatinina sérica dentro de 7 dias.

Apesar de as primeiras publicações acerca do uso de contraste iodado terem surgido em 1923, e seus efeitos adversos terem sido relatados em 1941, somente em 1954 surgiu o primeiro relato de caso de NCI. O paciente em questão era portador de mieloma múltiplo e desenvolveu IRA após ter sido submetido a uma pielografia intravenosa.

A utilidade de exames que utilizam o contraste iodado tem aumentado drasticamente. Dentre eles destaca-se o cateterismo cardíaco: em países ocidentais são realizados cerca de 8000 cateterismos cardíacos por milhão de habitantes. Nos EUA, houve um aumento de 390\% no número de intervenções cardíacas percutâneas entre 1979 e 2002.

Os métodos angiográficos representam inquestionáveis benefícios no diagnóstico, manejo e estratificação de risco em uma série de condições clínicas. No entanto, seu uso contribui para aproximadamente $12 \%$ das causas de IRA hospitalar devido aos efeitos do contraste radiológico, sendo a terceira causa de IRA iatrogênica em pacientes hospitalizados, aumentando o tempo de internação e morbi-mortalidade.

Estima-se que a incidência de NCI varie entre 1,6\% e 2,3\% na população em geral, porém essas taxas podem atingir níveis acima de $20 \%$ dependendo dos fatores de risco presentes, principalmente a presença de DM. De acordo com a OMS, a prevalência do DM no mundo em 2000 foi de 171 milhões, com expectativa que haverão 366 milhões de indivíduos diabéticos na população global em 2030. Sabe-se que a doença arterial coronariana ocorre com mais freqüência em diabéticos do que na população geral, afetando mais de $55 \%$ dos pacientes, sendo o DM fator de risco independente para doença cardiovascular, e justificando o grande emprego do cateterismo nesse grupo de pacientes. Outro componente agravante consiste na instalação insidiosa de lesão renal nesses pacientes, favorecendo ainda mais ao desenvolvimento de NCI.

Os mecanismos que levam à NCI são variados, e acredita-se que atuam de forma sinérgica. A combinação entre mecanismos de toxicidade direta provocada pelo contraste e de hipóxia induzem lesão renal.

Estudos realizados até a presente data não evidenciaram uma forma eficaz de tratar a NCI. 
No entanto, sabe-se que a melhor forma é evitá-la, utilizando medidas de prevenção. As mesmas, apesar de seus efeitos não serem $100 \%$ comprovados, se mostraram benéficas na redução no impacto causado pela NCI.

A elucidação dos mecanismos fisiopatológicos da NCI ainda permanece desconhecida, dificultando o desenvolvimento de métodos eficazes para sua prevenção e tratamento. Mais estudos são necessários no intuito de descobrir onde exatamente age o contraste iodado, e o que fazer para evitar que este agente aja de maneira imprópria na estrutura e no bom funcionamento renal.

Os esforços devem ser redobrados na população diabética. Esses pacientes invariavelmente desenvolvem nefropatia pela própria doença. Muitas vezes são também portadores de hipertensão arterial, como visto na presente pesquisa, condição também lesiva aos rins.

Os resultados obtidos na análise demonstram que a incidência média de NCI em diabéticos foi de 11 vezes maior que o encontrado na população geral, e portanto que existe forte associação do desenvolvimento de IRA induzida por NCI nesse grupo de pacientes devido à lesão renal preexistente, como comprovado por outros estudos.

A sobrevida da população em geral está crescendo cada vez mais, mesmo em pacientes diabéticos e hipertensos, devido a melhores condições de vida, medicamentos, hábitos saudáveis de alimentação, atividade física, e acesso à informação. No entanto, a doença coronariana acompanha o envelhecimento, e caminha lado a lado a comorbidades como o DM. A utilização de procedimentos de Hemodinâmica, expõe diabéticos ao somatório de diversos fatores de risco para o desenvolvimento de NCI, agravando sua condição renal.

\section{Conclusão}

Como visto nos resultados expostos, $21.05 \%$ dos pacientes diabéticos desenvolveram NCI, configurando incidência média 11 vezes maior quando comparado à população geral, portanto o uso do contraste iodado deve ser feito com parcimônia nessa população, e os procedimentos devem ser prescritos com bastante precisão, evitando assim a exposição desnecessária a este agente lesivo. 


\section{Referências}

Amini, M. et al. (2009) N-acetilciteine does not prevent contrast-induced nephropathy after cardiac catheterization in patients with diabetes mellitus and chronic kidney disease: a randomized clinical trial. Trials. no 10. Vol 45.

Barrett, B.J. et al. (1992) Contrast nephropathy in patients with impaired renal function: high versus low osmolar media. Kidney Int nº 41: 1274-9.

Buehler, A.M.; Berwagner. (2008) A N-acetilcisteína na prevenção da Nefropatia Induzida por Contraste: as Evidências são definitivas ou necessitamos de mais estudos? Rev Bras Cardiol Invas. no 2 vol. 16, pg 225 - 233.

Davidson, C.J. et al. (1989) Cardiovascular and renal toxicity of a nonionic radiographic contrast agent after cardiac catheterization: a prospective trial. Ann Intern Med n ${ }^{\circ} 110$, 119-24.

De Broe, M.E. Renal Injury Due to Enviromental Toxins, Drugs and Contrast Agents. Atlas of the Kidney Diseases. Livro 1; Cap 11, pg 15.

Delia, J.A. et al. (1982) Nephrotoxicity from angiographic contrast material. A prospective study. Am J Med no 72: 719-25.

Diogo, L.P. et al. (2008) Existe associação entre antiinflamatórios não-esteróides e nefropatia induzida por contraste? Rev Scien Med. no 3 vol 18, pg 133 -140.

Efstratiadis, G. et al. (2008) Contrast media-induced nephropathy: case report and review of the literature focusing on pathogenesis. Hippokratia. no 2 vol. 12, pg 87 - 93.

Gomes, V.O. et al. (2008) Hidratação com bicarbonato de sódio na prevenção de nefropatia induzida por contraste em pacientes diabéticos: Subanálise de ensaio clínico multicêntrico. Rev Bras Cardiol Invas. no 4. Vol 16; pg 434-436.

Gus, I. et al. (2002) Prevalência dos Fatores de Risco da Doença Arterial Coronariana no Estado do Rio Grande do Sul. Arq Bras Cardiol. no 78, pg 478 - 483.

Kelly, A.M. et al. (2008) Meta-analysis: Effectiveness of Drugs for Preventing ContrastInduced Nephropathy. An Int Med. no 148, pg 284 - 294.

Kidney Disease Outcome Quality Initiative: KDOQI. Clinical Practice Guidelines and Clinical Practice Recommendations for Diabetes and Chronic Kidney Disease. New York: National Kidney Foundation (2007). Acessado em http://www.kidney.org/ professionals/kdoqi em 11 de Dezembro de 2009.

Kramer, C.K. et al. (2008) Nefropatia induzida por contraste: medidas de prevenção. Rev HCPA. no 28. Vol.1; pg 33 - 36.

Lautin, E.M. et al. (1991) Radiocontrast-associated renal dysfunction: incidence and risk factors. Am J Roentgenol nº 157: 49-58.

Macedo, E. et al. (2006) Lack of protection of N-acetylcysteine (NAC) in acute renal failure related to elective aortic aneurysm repair-a randomized controlled trial. Nephrol Dial Transplant no. 21, pg 1863 - 1869.

Machado, M.C. et al. (2003) Nefropatia por Radiocontraste: Tendências Atuais. Rev Brasil Cardiol Invas. no 4 vol. 11; pg 27 - 30. 
Marenzi, G. et al. (2006) N-Acetylcysteine and Contrast-Induced Nephropathy in Primary Angioplasty. N Engl J Med no. 354, pg 2773 - 2782.

Marenzi, G. et al. (2009) Contrast Volume During Primary Percutaneous Coronary Intervention and Subsequent Contrast-Induced Nephropathy and Mortality. Ann Intern Med no.150, pg 170 - 177.

McCullough, P.A. et al. (1997) Acute renal failure after coronary intervention: incidence, risk factors, and relationship to mortality. Am J Med nº 103: 368-75.

Mehran, R. et al. (2004) A simple risk score for prediction of contrast-induced nephropathy after percutaneous coronary intervention: Development and initial validation. J Am Col Cardiol. no. 44, pg 1393 - 1399.

Murphy, S.W. et al. (2000) Contrast Nephropathy. J Am Soc Nephrol. no 111, pg 177182.

Parfrey, P.S. et al. (1989)Contrast material-induced renal failure in patients with diabetes mellitus, renal insufficiency, or both: a prospective controlled study. N Engl J Med $\mathrm{n}^{\circ}$ 320: $143-9$.

Paula, A.S. et al. (2006) Efeito protetor da N-Acetilcisteína em pacientes com função renal normal expostos a contraste radiológico hiperosmolar. Jorn Bras Nefrol. no 28. vol.1; pg 15 - 19.

Pereira, B.J. et al. (2001) Nefrotoxicidade por contraste radiológico iodado endovenoso: o que há de novo. Rev Diag e Tratam. no 2 vol. 6, pg 29 - 33.

Recio-Mayoral, A. et al. (2007) The Reno-Protective Effect of Hydration with Sodium Bicarbonate Plus N-Acetylcysteine in Patients Undergoing Emergency Percutaneous Coronary Intervention: The RENO Study. J Am Coll Cardiol no. 49, pg 1283-1288.

Rudnick, M.R. et al. (1995) Nephrotoxicity of ionic and nonionic contrast media in 1196 patients: a randomized trial: the Iohexol Cooperative Study. Kidney Int $n^{\circ}$ 47: 25461.

Solomon, R. (2009) Preventing contrast-induced nephropathy: problems, challenges and future directions. BMC Medicine. Vol. 7,pg 24 - 28.

Weisberg, L.S. et al. (1994) Risk of radiocontrast nephropathy in patients with and without diabetes mellitus. Kidney Int $n^{\circ} 45,259-65$.

World Health Organization. Acessado em http://www.who.int/diabetes no dia 14 de Dezembro de 2009 às 14:50h. 